\section{P390 IMPROVING THE UPTAKE OF HIV-CENTERED PREVENTATIVE AND TREATMENT SERVICES BY ADOLESCENTS}

Chantal Smith*. MaCH, Health Systems Strengthening, Durban, South Africa

10.1136/sextrans-2019-sti.484

Background There is approximately 6.3million people living with HIV in South Africa with nearly a third of all new HIV infections occurring in 15-24 year olds with adolescent girls being up to eight times more likely to be infected with HIV than their male counterparts. In addition, 52 percent of all sexually active young people who reside in rural areas have reported having never used condoms with their most recent partners.

Methods At facility-level, MatCH deployed a roving clinical team, consisting of clinical and a psychosocial advisors, who provided the following: (a) onsite training and mentoring of healthcare workers on the delivery of adolescent friendly services, (b) identification of healthcare workers as Adolescent Champions, (c) developing dedicated consultation rooms for adolescents to seek clinical and psychosocial support and to be fast-tracked for immediate management. At patient-level, $\mathrm{MatCH}$ designed an HIV/STI preventative package of care inclusive of: HIV testing services, behavioural risk assessments, PrEP initiation for at-risk HIV-negative adolescents, STI, gender-based violence and TB screening and management, sameday ART initiation for HIV-positive adolescents and ongoing psychosocial support via one-on-one adherence counselling and linking to peer-led support groups.

Results Comparative review of 8 -month data, revealed a $70 \%$ increase in the number of adolescents accessing HIV testing services at PHC facilities. Of those who tested HIV-positive, linkage to care improved by $98 \%$ as compared to the baseline of 63\%. 90\% uptake of PrEP by at-risk HIV negative females aged 15-19years as compared to the $10 \%$ uptake seen amongst their male counterparts within the same age group. Improved healthcare worker confidence in (a) managing at-risk HIV negative adolescents, (b) initiating HIV positive onto ART and (c) delivering comprehensive package of HIV-centered adolescent friendly services.

Conclusion The provision of adolescent-friendlyservices at primary healthcare facilities is essential to improving the uptake of HIV-centered prevention and treatment strategies by at-risk adolescents.

Disclosure No significant relationships.

\section{P391 YOUNG PEOPLE'S EXPERIENCES WITH CLINICAL CARE AND PREFERENCES FOR DELIVERY OF NEW POINT-OF- CARE TESTS FOR STIS IN ENGLAND}

${ }^{1}$ Hannah Mcculloch, ${ }^{2}$ Agata Pacho, ${ }^{1}$ Rebecca Geary, ${ }^{2}$ Sebastian Fuller*. ${ }^{1}$ London School of Hygiene and Tropical Medicine, Faculty of Public Health and Policy, Department of Health Services Research and Policy, London, UK; ${ }^{2}$ St George's, University of London, Applied Diagnostic Research and Evaluation Unit, Institute for Infection and Immunity, London, UK

\subsection{6/sextrans-2019-sti.485}

Background Young people in the UK face multiple barriers to attending sexual health clinics (SHCs) and are at high risk for sexually transmitted infections (STIs), despite widely available free and confidential STI testing. Studies have shown that point-of-care tests (POCTs) for STIs, which can accurately diagnose and treat patients in one clinical visit, have potential to change clinical practice. It has been recommended that young people be consulted in advance of changes to care.

Methods We conducted a secondary analysis of qualitative indepth interviews with young participants of the Precise study, which explored patient experiences of SHC services and their opinions of POCT implementation plans. Attendees were purposively sampled at three SHCs in England. Interviews were thematically analysed using NVivo (V10) to investigate young people's perceptions of their healthcare needs and preferences for POCTs.

Results Ten young people aged 17-22 years, including: three women-who-have-sex-with-men, four heterosexual men and three men-who-have-sex-with-men were interviewed between June 2015 and February 2016. Participants generally regarded the tests as innovations to improve SHC experiences including the potential to reduce time-to-results and receive results from trusted sexual health experts. Participants raised concerns that POCT implementation may shorten consultation times and changes to pathways may unintentionally impact on privacy and/or cause confusion. Participants suggested increase in provision of information to patients, including potential waiting times and self-sampling instructions, along with recommendations to safeguard privacy around sample provision and clinical consultation times.

Conclusion Many of our young participants discussed waiting for results to be anxiety-producing, with provision of POCTs mitigating this concern, increasing satisfaction with SHC care and potentially paving the way for increased attendance among this group. However, participants suggest that other barriers, specifically around confusion and privacy, are likely to mitigate positive effects if not addressed by appropriate clinical implementation plans.

Disclosure No significant relationships.

\section{P392 TEENS' EXPERIENCES FOLLOWING SEXUAL ASSAULT: A SYSTEMATIC REVIEW OF QUALITATIVE RESEARCH}

${ }^{1}$ Tamar Shiboleth, ${ }^{2}$ Annalise Weckesser, ${ }^{3}$ Jonathan Ross*, ${ }^{3}$ Rachel Caswell. ${ }^{1}$ Birmingham City University, Hels, Birmingham, UK; ${ }^{2}$ Birmingham City University, Hels, Birmingham, UK; ${ }^{3}$ University Hospital Birmingham NHS Trust, Birmingham, UK

\subsection{6/sextrans-2019-sti.486}

Background Data shows adolescents have the highest rate of sexual assault. Adolescents who were raped during childhood show a relation with risky behaviours such as being sexually active at a younger age and poor use of contraception. Furthermore, they have a greater number of pregnancies and abortions, are at higher risk for HIV and STI's, higher rates of depression and suicidal thoughts and attempts, self-mutilation, eating disorders and obesity (Crawford-Jakubiak, Alderman \& Leventhal, 2017; Fier, 2017; Brabant, Hébert \& Chagnon, 2013; Keeshin et al., 2013).

Adolescents appear to have special needs when it comes to sexual health care services (Kulyk et al., 2013). Even though the emotional, physical, and social consequences of sexual assault are similar to those of adults, 'adolescents have less life experience and emotional fortitude to handle the trauma' (Bein, 2011) associated with sexual assault. Even so, they have proved hard to reach by health and support services.

A review of the literature shows few studies actually focus on adolescents' experiences and needs after sexual assault. The current study is part of a larger (doctoral) study into teens' 